\title{
Post-traumatic inflammation following spinal cord injury
}

\author{
ON Hausmann*,1,2 \\ ${ }^{1}$ Neurosurgical Department, University Clinics Basel, Switzerland; ${ }^{2}$ Neurosurgery Clinic St Anna, Lucerne, Switzerland
}

Inflammatory reaction following a spinal cord injury (SCI) contributes substantially to secondary effects, with both beneficial and devastating effects. This review summarizes the current knowledge concerning the structural features (vascular, cellular, and biochemical events) of SCI and gives an overview of the regulation of post-traumatic inflammation.

Spinal Cord (2003) 41, 369-378. doi:10.1038/sj.sc.3101483

Keywords: spinal cord injury; inflammation; microglia; macrophages; astrocytes

\section{Introduction}

Spinal cord injury (SCI) initiates a robust immune response characterized in part by the synthesis of cytokines and chemokines and a coordinated infiltration of the damaged site by peripheral leucocytes. ${ }^{1-8}$ SCIinduced inflammation may result in further reduction in functional recovery because of the development of scar tissue, as well as necrosis or apoptosis of neurons and oligodendrocytes. However, a potentially beneficial role of the inflammatory process has also been reported, illustrating the dual nature of post-traumatic inflammation. ${ }^{6}$ The exact mechanism and consequences of a SCIinduced inflammatory response, with activation of central nervous system (CNS) resident microglia and recruitment of blood-born inflammatory cells, are not fully understood at present.

Within the context of modulation of the posttraumatic events, this review focuses on the regulation of the inflammatory response following SCI. Compared to an equivalent stimulus in peripheral non-neuronal tissue, the inflammatory response seen in the CNS is generally much weaker. ${ }^{9,10}$ Several unique characteristics of CNS tissue contribute to CNS-specific responses to trauma. First, the blood-brain barrier (BBB) largely excludes serum proteins from the extracellular CNS milieu. Further, microglia, the CNS resident macrophages, are normally in a quiescent state and must be activated to evoke a response. ${ }^{9}$ The CNS extracellular matrix consists of large molecular weight proteins (such as laminin, collagen, fibronectin, proteoglycan, and tenascin) that are known to regulate cell behaviour during development and in response to injury. SCI induces synthesis of tenascin- $\mathrm{C}$, keratan sulphate and

*Correspondence: ON Hausmann, Klinik St Anna, St Anna-Strasse 32, 6006 Lucerne, Switzerland chondroitin sulphate proteoglycans (CSPs) by reactive microglia, macrophages, and oligodendrocytes. ${ }^{11-13,112}$ However, many of the molecules necessary for initiation and modulation of the inflammatory response are present in the CNS parenchyma. For example, chemokines and cytokines are expressed in the CNS either constitutively or in response to injury. ${ }^{14}$ The infiltration of the CNS by leucocytes is orchestrated by specific cell adhesion molecules that promote cell-to-cell interactions between astrocytes, endothelial cells, microglia, and macrophages. ${ }^{3}$ Interestingly, it has been shown that the acute inflammatory response to traumatic injury is greater in the spinal cord than in the cerebral cortex. ${ }^{9,10}$ Following a comparable mechanical injury, the number of neutrophils and activated microglia/macrophage, as well as the extent of their infiltration, was much less in the cerebral cortex than in the spinal cord. ${ }^{9}$ However, in contrast to the less permissive environment of the cerebral cortex, a marked inflammatory reaction in the meninges and the choriod plexus may be seen. Finally, the absence of mast cells and dendritic cells contributes to the atypical characteristics of the inflammatory response in the CNS.

While initial damage is induced by contusion of the cord (for example, haemorrhage, membrane disruption, and vascular damage), the final pathohistological lesion is far greater than that identifiable in the first few hours after the injury. Clearly, the size of the final lesion is correlated to functional outcome. The spread of the damage (such as excitatory amino-acid-induced intracellular $\mathrm{Ca}^{2+}$ increase, proteolysis, formation of free radicals and nitric oxide) is thought to be because of the activation of the biochemical events leading to cellular dysfunction or even cellular death. ${ }^{15-18}$ This cascade of injury-induced, destructive events is defined as 
secondary injury. Another important characteristic of SCI is that longitudinal spreading of the secondary injury causes further segmental loss of function.

It is important to point out that regulation of the secondary injury cascade may differ significantly between animal strains and species. Consequently, promising scientific observations or potential therapies may not be interspecific or even interstrain applicable without appropriate investigation in the respective animal model. However, one feature of the post-traumatic reaction of the CNS that occurs regardless of species, animal strain, or injury model, is the patterned and regional degeneration of both myelinated and unmyelinated axons. This is accompanied by local activation of resident microglia cells. ${ }^{14}$

\section{Primary and secondary injury}

The SCI site contains large numbers of both apoptotic and necrotic neurons and glia. Typically, the centre of a SCI is predominantly characterized by necrotic death. The primary injury refers to the mechanical damage leading to direct cell death and bleeding. Further progressive destruction of the tissue surrounding the necrotic core is known as secondary injury. ${ }^{8,19}$ There are parallels between this area of secondary tissue damage and damage seen in the penumbra zone following a focal ischaemic lesion in which apoptotic cell death also dominates. Several mechanisms contribute to this pattern of destruction including hypoxia, excitotoxicity, free radical formation, release of proteases, inflammatory response with activation of CNS resident microglia and invasion of peripheral macrophages. Table 1 summarizes the cascade of vascular, cellular, and biochemical events of the secondary neuroinjury.

Table 1 Summary of events of secondary neuroiniury following SCI

Vascular events: breakdown of blood-spinal cord barrier oedema formation ischaemia and hypoxia release of vasoactive substances alteration of spinal cord perfusion

Biochemical events:excitotoxicity formation of free radicals and nitric oxide release of proteases damage of mitochondrias energy depletation

Cellular events: invasion of neutrophils activation of resident microglia invasion of peripheral macrophages infiltration of lymphocytes activation of astrocytes apoptosis of oligodendrocytes Wallerian degeneration

\section{Experimental models}

In general, to study the pathophysiology of SCI on the cellular and molecular levels, animal models of SCI have to be reliable, consistent, and reproducible. To have clinical relevancy, they need to imitate the pathological features of human SCI. In most laboratories, either contusion injury or transection models are used in mammals such as rats or mice. The standardized NYU weight drop contusion model allows the induction of reproducible minor, moderate, or severe contusion injury typically of the thoracic spinal cord. ${ }^{20-23}$ Other laboratories use electromechanical-driven, feedbackcontrolled impaction devices (for example, the Ohio State or OSU device). ${ }^{24,25}$ Further, experimental injuries using an extradural clip compression at the cervical level have been reported. ${ }^{26}$ Intraspinal injection of quisqualic acid (QUIS) produces excitotoxic injury with pathophysiological characteristics similar to those associated with traumatic SCI. ${ }^{27,28}$

To take advantage of various transgenic and knockout mice, murine SCI models using dorsal hemisection ${ }^{29}$ or extradural calibrated clip compression have recently been used with a modified murine BBB hind limb locomotor rating scale. ${ }^{30,31}$ The histopathology correlates positively with the biomechanical impact. ${ }^{32}$

\section{Lesion cavity}

Typically, the contusion SCI model is characterized by a central haemorrhagic necrosis that spreads radially and rostrocaudally, resulting in an ellipsoidal, loculated cystic cavity. The cavity is filled with macrophages and lined by activated astrocytes. Cells born in the rostral and caudal ependymal zones contribute to trabeculae that divide the cavity and provide a matrix for invading Schwann cells and axons. ${ }^{15}$ A peripheral rim of spared axons persists in the ventral and dorsal funiculi that may be seen even after severe injuries. The centre of the cavity is filled with granular debris and fascicles of small myelinated and unmyelinated axons are interspersed with macrophages. Large numbers of activated resident microglia and invading peripheral macrophages are present soon after the injury and persist for several months. The area is occupied by granular debris, myelin fragments, macrophages, and invading blood vessels. The amount of spared white matter in the thoracic spinal cord correlates highly with preserved locomotor function. ${ }^{17,21,33,8,110}$ Pharmaceutical treatment in the acute phase following SCI, targets such spared tissue to reduce the spread of secondary injury, leaving more fibres and myelin in the white matter intact. Fibres can be seen approaching the rostral end of the lesion and branching into the adjacent grey matter. ${ }^{15}$ Many degenerating axons exhibit retraction bulbs. ${ }^{34-36}$

In contrast to the contusion model, transection lesions are characterized by a limited rostrocaudal spread of secondary injury. ${ }^{15}$ Transections or hemisections of the spinal cord include damage to the meninges with consequent differences in the invasion by peripheral macrophages and meningeal cells. 
Vascular events of secondary injury

Vascular events of secondary injury include the disruption of the blood-spinal cord barrier (BSB) that is closely associated with oedema formation. Breakdown of the BSB also triggers the post-traumatic inflammatory response by invasion of neutrophils and macrophages. Further, trauma-activated endothelial and glial cells release vasoactive substances (eg reactive oxygen molecules, bradykinins, histamines, and nitric oxide) that influence the spinal cord perfusion and facilitate the crossing of plasma-derived molecules into the cord. ${ }^{9}$ Together, these vascular events, being part of the secondary injury cascade, play an essential role in activating and regulating the secondary events, including the post-traumatic inflammatory response. The disruption of the BSB is characterized both by a transient loss of anionic charge at sites along the endothelial glycocalyx and extravasation of plasma proteins, including cytokines. ${ }^{37}$ The BSB breakdown is maximal 1 day after lesion and gradually declines thereafter. ${ }^{9,38}$ BSB breakdown extends along the axis of the injured spinal cord and consequently is not restricted to the injured site. ${ }^{7,9,37,39}$ However, the area and duration of increased membrane permeability is much greater in the spinal cord than in the brain following a comparable lesion. ${ }^{9}$

Tator and Koyanagi ${ }^{40}$ report in their study on the vascular effects in human SCI, that at the level of the injury, haemorrhagic patterns were more pronounced in the grey matter. Conversely, most of the white matter showed nonhaemorrhagic degenerative changes including myelin degradation and axonal swelling in the acute stage. ${ }^{40}$ Since at the site of injury, none of the major arteries on the surface of the spinal cord was found to be occluded, the authors have suggested that the intramedullary vascular system may be primarily responsible for the vascular damage seen in humans.

Liberation of catalytic metal ions, which are present in high concentration during the degradation of haemoglobin, is essential to the formation of free radicals. ${ }^{39}$ Ischaemic tissue exhibits profound energy depletion by loss of adenosine triphosphate (ATP). Restoration of ATP pools in sublethally ischaemic cells causes some cells to undergo reperfusion injury because of reoxygenation. This leads finally to energy-dependent apoptosis. ${ }^{41}$ These questions concerning energy depletion will be addressed later.

\section{Biochemical events of secondary injury}

$\mathrm{Ca}^{2+}$-dependent, glutamate-associated neuronal cell death SCI initiates biochemical cascades that lead to an increase in extracellular excitatory amino-acid (EAA) concentration, resulting in glutamate receptor-mediated excitatoxic events. ${ }^{42}$ Glutamate is a major excitatory neurotransmitter in the mammalian nervous system. After its release, specific transporter proteins rapidly remove extracellular glutamate from the synaptic cleft. The clearance of excess glutamate prevents accumulation under normal conditions. However, SCI elevates extracellular glutamate concentrations to neurotoxic levels. Excitotoxicity refers to the ability of glutamate and aspartate to destroy neurons by prolonged excitatory synaptic transmission. Glutamate cell membrane receptors can be divided either into the (i) ionotropic $N$ methyl-D-aspartate (NMDA) and AMPA/kainate receptors or (ii) the metobotropic glutamate receptors. ${ }^{43,44}$ However, there is general consensus that the NMDA glutamate receptor subtype plays a key role in mediating certain aspects of glutamate excitotoxicity in SCI. ${ }^{27,34,45,46}$ Expression of glial and neuronal glutamate transporter proteins is upregulated rapidly after SCI, reaching peak values within $6 \mathrm{~h}$, decreasing thereafter, but remaining elevated for $24 \mathrm{~h} .{ }^{47}$ Further, the three different subtypes of metabotropic glutamate have different spatial and temporal expression patterns following SCI ${ }^{42,44}$ Since metabotropic glutamate receptors are G-protein coupled, their activation can initiate numerous intracellular signalling pathways that have a variety of complex and long-lasting effects following SCI. ${ }^{44}$ Interestingly, spinal neurons were found to be less vulnerable to NMDA toxicity and more vulnerable to AMPA/kainate toxicity than cortical neurons. ${ }^{48}$ In general, hypoxia makes cells more sensitive to glutamate challenge. ${ }^{4}$

In the adult mammalian nervous system, cellular $\mathrm{Ca}^{2+}$ overload is intimately related to glutamateinduced traumatic and ischaemic neuronal cell death, both of which are associated with increased tissue $\mathrm{Ca}^{2+}{ }^{45,50}$ Recent work suggests that both the total neuronal $\mathrm{Ca}^{2+}$ load and the route by which the loading occurs to be important. ${ }^{45}$ The influx of $\mathrm{Ca}^{2+}$ ions into neurons by glutamate activation of NMDA receptors leads to activation of various secondary processes. This leads to cellular death. EAA induce a prompt intracellular $\mathrm{Ca}^{2+}$ increase. This triggers the $\mathrm{Ca}^{2+}$-dependent neurotoxicity at a very early time point.

$\mathrm{Ca}^{2+}$ excess-related neurotoxicity is responsible for further specific processes including formation of free radicals and nitric oxide, activation of proteases and endonucleases, mitochondrial damage, and induction of apoptosis. These will be discussed in the following sections.

Formation of free radicals and nitric oxide Free radicals such as superoxide $\left(\mathrm{O}_{2}^{-}\right)$and hydroxyl $\left(\mathrm{OH}^{-}\right)$are produced in small amounts by normal cellular processes as part of the mitochondrial electron transport chain and the microsomal cytochrome $P-450$ system. Free radicals play important physiological roles such as modulation of membrane receptor function. For example, free radicals are known to affect NMDA function. ${ }^{51}$

Free radicals are formed during traumatic or hypoxic injuries as a consequence of insufficient oxygenation. Further, during post-traumatic reperfusion, reactive oxygen radicals may also be formed as byproducts of the biochemical reactions, which produce prostaglandins and leucotrienes from arachidonic acid. ${ }^{8,45,52}$ Liberation of catalytic metal ions, that are present in 
high concentration during the degradation of haemoglobin, is essential to the formation of free radicals.

Free radicals can react with and subsequently damage proteins, nucleic acids, lipids, and extracellular matrix proteins such as glycosaminoglycans. Polyunsaturated fatty acids are found in very high concentrations in the CNS as plasma membrane components. They are particularly vulnerable to free radicals and this vulnerability is responsible for the deleterious post-traumatic ischaemia-induced lipid peroxidation within the CNS.

Nitric oxide (NO) is a diffusible highly reactive gas that is produced physiologically in small amounts in the $\mathrm{CNS}$ by the vascular endothelium and neurons. NO acts as a potent vasodilatator. ${ }^{53} \mathrm{NO}$ per se is not highly toxic. However, it reacts with $\mathrm{O}_{2}^{-}$to form the powerful oxidant, peroxynitrite $\left(\mathrm{ONOO}^{-}\right)$that can directly oxidize lipids, DNA, and proteins. ${ }^{54}$ Immediately following contusion SCI, concentrations of NO markedly increase and then gradually decrease between 1 and 12 days after injury. ${ }^{55} \mathrm{ONOO}^{-}$itself causes significant neuronal loss and locomotor dysfunction when generated in the rat spinal cord in vivo. ${ }^{56}$ The endogenous free radical scavenger, superoxide dismutase (SOD) can moderate this reaction. Excessive NO production has been postulated to be the causative mechanism underlying neurotoxicity. 57

\section{Mitochondrial damage}

A landmark of secondary tissue loss following CNS injury is the devasting loss of intracellular energy substrates. $^{33,58,59}$ This energy loss is caused by vascular damage and subsequent reperfusion-induced endothelium damage. ${ }^{40,60}$ Hypoxia causes dysfunction of mitochondria, which normally serve as an energy buffer during physiological and pathological conditions. However, mitochondrial dysfunction is a common event in cell injury in ischaemic or mechanical injury. This may be caused by the anoxic collapse of the transmitochondrial membrane potential leading to intramitochondrial $\mathrm{Ca}^{2+}$ accumulation. It is likely that extreme intracellular $\mathrm{Ca}^{2+}$ overload irreversibly damages mitochondria and ATP depletion leading the cell to die. ${ }^{41}$

\section{Cellular reaction of secondary injury}

Invasion of neutrophils Neutrophils are the first inflammatory cells to arrive at the site of injury in nonneuronal and neuronal tissue. By their phagocytic and properties, they are able to remove tissue debris and restore homeostasis. Post-traumatic accumulation of neutrophils, as measured by myeloperoxidase (MPO) activity, is significantly increased within $3 \mathrm{~h}$ and remains elevated up to 3 days after SCI. ${ }^{61-63}$ Typically, neutrophils accumulate at the vascular endothelium. Although neutrophils accumulate also intraparenchymally in areas adjacent to the haemorrhagic necrosis, there is no histological evidence that they infiltrate the spinal cord. ${ }^{61,63}$ Compared to an experimental brain injury, the number of recruited and infiltrated neutro- phils is much higher in the spinal cord following a similar lesion. ${ }^{9}$ The greater degree of neutrophil recruitment in the spinal cord may be because of the stronger upregulation of intercellular adhesion molecule (ICAM-1) and platelet-endothelial cell adhesion molecule (PECAM). Both molecules are expressed in endothelial cells. However, higher amounts are found in the cord as compared to the cerebral cortex. ${ }^{10}$ Neutrophils are involved in the modulation of the secondary injury by release of neutrophil proteases and reactive oxygen species. Neutrophil elastase is an enzyme capable of damaging endothelial cells resulting in increased vascular permeability. Since post-traumatic haemorrhage within the spinal cord is markedly reduced by an neutrophil elastase inhibitor, haemorrhage may be a consequence of neutrophil elastase-induced endothelial cell damage. ${ }^{60}$

Microglia activation and invasion of macrophages Resting microglia occupy approximately $13 \%$ of the entire glial cell population and are distributed diffusely throughout the CNS. ${ }^{64}$ Microglia respond rapidly to disturbances within the microenvironment by change in morphology, expression of specific cell surface molecules, and release of cytokines such as interleukin-1 (IL$1)$, tumour necrosis factor alpha (TNF- $\alpha)$ and chemokines such as leucotrienes and prostaglandins. The term 'activated microglia' is used to describe proliferating nonphagocytic cells that demonstrate changes in their immunophenotype and their morphology, but have not undergone transformation into brain phagocytic macrophages. 7,65

However, it is not possible to reliably distinguish activated microglia from invading peripheral bloodborn macrophages using immunohistochemistry alone. The morphological diversity of microglia is indicative of functional heterogeneity. 2,36,64,65 For example, the concomitant increase in expression of major histocompatibility complex (MHC) antigen class II is only seen in a subpopulation of microglia with increased OX42 immunoreactivity. ${ }^{7}$ The OX42 antibody recognizes complement CD 11 (C3b receptor) expressed by resting and activated resident microglia as well as by peripheral macrophages. ${ }^{61}$ Microglia/macrophages are the only OX42-positive cells present in the CNS. The ED1 antibody reacts with an intracellular antigen in activated microglia and macrophages. Resting microglia are recognized by their distinct star-shaped morphology, particularly with the OX42 antibody. Conversely, activated phagocytotic microglia are rounded and, are therefore, not easily distinguishable from peripheral macrophages. ${ }^{61}$ Interestingly, in contrast to ED1-positive phagocytic macrophages, activated microglia were found distal to the lesion centre and in areas with moderate rather than severe demyelination. ${ }^{7}$ In the first $24 \mathrm{~h}$ following transection of the spinal cord, a narrow belt containing a dramatic reduction in the number of OX42-positive microglia has been described around the injury site. ${ }^{64}$ 
Post-traumatic activation of microglia is evident at day one. However, the number of activated microglia increases during the first 7 days and then plateaus, 2-4 weeks postinjury, depending on the animal strain. ${ }^{7}$ Thus, the mononuclear phagocytic response to SCI is considerably greater than that seen following a similarsize lesion in the forebrain. ${ }^{9}$

It is well known that activated microglia secrete cytotoxic substances including various cytokines such as TNF- $\alpha$, IL-1, reactive free radicals, and nitric oxide. However, the major role of microglia at the lesion centre is probably rapid phagocytosis of debris rather than induction of apoptosis. ${ }^{66}$ Heterogeneous neurotrophin expression in vivo and differential responses to neutrotrophins by microglia in vitro suggest that these cells may elicit unique functional properties in the pathological CNS and thus, subsequently control the inflammatory response at the injury site. ${ }^{14}$

The prolonged presence of active microglia/macrophages in CNS tissue, in contrast to the peripheral nervous system, has a number of effects, either deleterious or beneficial. ${ }^{8,67}$ For example, prolonged release of proinflammatory cytokines by microglia/ macrophages may contribute to subsequent further destruction. On the other hand, activation of microglia as well as astrocytes may lead to the production of growth factors essential for neuronal survival and tissue repair. Further, results have suggested that transplantation of peripherally activated macrophages has beneficial effects on functional spinal cord regeneration. Clearly, the environment dictates the response of the macrophage. ${ }^{68}$

Lymphocyte infiltration T-lymphocytes are scattered in the uninjured spinal cord and progressively increase in number after injury in parallel with the activation of microglia and influx of peripheral macrophages, within the first week and predominantly within the epicentre. ${ }^{14}$ Under normal conditions, activated $T$ cells can cross the $\mathrm{BBB}$ and enter the CNS parenchyma. In comparison with other inflammatory cells recruited, the number of lymphocytes remains low. ${ }^{9}$ However, T-lymphocytes play an important role in the CNS immune system, since on activation, T-lymphocytes may kill target cells and produce cytokines.

Schwartz and co-workers have pointed out that both innate and adaptive autoimmune responses may be needed for recovery after axonal CNS injury; that macrophages are required for repair, and that activated $\mathrm{T}$ cells directed against CNS antigenes are needed for defence and protection. ${ }^{67} \mathrm{~A}$ single postinjury injection of autoimmune $\mathrm{T}$ cells directed against CNS myelin basic protein has been found to reduce the spread of damage and promote recovery following contusion SCI in rats. ${ }^{69}$ The degree of functional recovery was found to be dependent on the final lesion as revealed through morphometric analysis, immunohistochemical staining, and techniques of diffussion magnetic resonance imaging. ${ }^{70}$ Collectively, these studies suggest that the slow longitudinal degeneration seen within the spinal cord because of axoplasmatic disruption following SCI may have consequences that are both advantages and deleterious to functional outcome. Degeneration then may be considered to have dual action and should not necessarily be regarded as an undesirable process. ${ }^{67,113}$

Astrocytic activation/GFAP upregulation After SCI, a selected population of astrocytes known as reactive astrocytes, contributes to the inhibitory environment within the injured spinal cord. Typically in mammals, there is a subsequent proliferation and hypertrophy of astrocytes around the injury site. ${ }^{8,9,48,71}$. Specifically, reactive astrogliosis is denoted by increased immunoreactivity of glial fibrillary acid protein (GFAP), which is a distinct cellular marker of astrocytes. ${ }^{14,48,71-74,111} \mathrm{It}$ has been demonstrated that reactive adult astrocytes upregulate the production of laminin and neurotrophin C. ${ }^{75,76}$ In addition to the endogenously present myelinassociated neurite growth inhibitory constitutents, reactive astrocytes form an astroglial scar that acts as a physical and/or chemical barrier to axonal regeneration. $8,35,72,77,78$ Proximity to the lesion epicentre determines whether the reactive glial environment will be growth supportive or inhibitory. ${ }^{63}$ Although the functional role of glial scarring is not completely understood, it has been suggested to be an attempt by the CNS to restore homeostasis through isolation of the damaged region. ${ }^{71,74,79}$ Activated astrocytes upregulate expression of various cell surface molecules including cell adhesion molecules and extracellular matrix proteins.

Apoptosis Apoptosis permits cell death in the absence of an inflammatory response in the surrounding tissue. Apoptosis is fundamentally distinct from necrosis; characterized by initial surface blebs, cell shrinkage, chromatin aggregation with genomic fragmentation, and nuclear pyknosis. ${ }^{16,80}$ In contrast, necrosis is characterized by passive cell swelling, mitochondrial damage with rapid energy loss, and disruption of internal homeostasis. ${ }^{81}$ The sequence of events leading to membrane rupture with release of intracellular constitutes activates a rapid inflammatory reaction.

Apoptosis is regulated through numerous genes, some of which have remained highly conserved throughout evolution. The mammalian apoptotic cell death programme is regulated by the caspase family of cysteine proteases. ${ }^{16,41,80,82}$ In particular, downstream caspase-3 has been shown to be important to neuronal development and injury by inducing fragmentation of nuclear DNA in vitro and in vivo. ${ }^{83,114}$ Additional control of cell death/survival is provided by the mammalian antiapoptotic proteins $\mathrm{Bcl}-\mathrm{X}_{\mathrm{L}}$ and $\mathrm{Bcl}-2$. The latter was shown to be upregulated in injured white matter axons following SCI. Major proapoptotic proteins are Bax, Bad, and Bid. 
Two different apoptosis signalling pathways in mammalian cells have been described. The first intrinsic pathway is initiated by mitochondrial dysfunction. Mitochondrial dysfunction can be caused by various triggers such as hypoxia or ATP depletion, which results in cytochrome $c$ release from mitochondria and triggering of caspase- 9 activation. The intrinsic pathway is mainly modulated by Bcl-2 and Bax, which are anti- and proapoptotic proteins, respectively. ${ }^{41}$ The second wellestablished apoptosis pathway involves signalling by cell surface 'death receptors'. These 'death receptors' are members of the TNF receptor (TNFR) family and include TNFR1, Fas, Fas ligand, p75, and DR3. These receptors recruit and activate caspase- 8 and caspase- 10 through their intracellular death domains. ${ }^{26,84-86}$ Fas and p75 receptors are expressed on oligodendrocytes, ${ }^{29}$ astrocytes, and microglia in the spinal cord following SCI. ${ }^{26}$ NMDA receptor activation by EAA, such as glutamate, promotes delayed apoptotic neuronal and glial cell death. The exact mechanism by which EAA induce apoptosis remains unclear. However, $\mathrm{Ca}^{2+}$ dependent and/or TNF- $\alpha$-mediated activation, in part via NO produced inducible NO synthase (iNOS), may be involved ${ }^{57,87}$ Further, it has been demonstrated that TNF- $\alpha$ potentiates cell death mediated by glutamate. ${ }^{29}$ Activation of nuclear factor kappaB (NF- $\kappa$ B) by TNF- $\alpha$ induces transcriptional activation of iNOS, thus causing further cell damage. ${ }^{3}$ This effect is partially blocked by the NMDA receptor antagonist MK-801 in the spinal cord white and grey matter and offers a potential therapeutic target. $^{88}$

In the CNS, apoptosis involves predominantly nonneuronal cells such as oligodendrocytes. ${ }^{16,26,66}$ Apoptotic cells were observed, mainly in the grey matter, $1 \mathrm{~h}$ after trauma with an increase for the first $8 \mathrm{~h}$, at which time the cells were found both in the grey and white matter. ${ }^{86}$ Interestingly, from 24 to $72 \mathrm{~h}$ postinjury, the number of apoptotic cells decreased in the grey matter, but increased in the white matter. ${ }^{86}$ Apoptotic cells are greatest in number closest to the lesion epicentre and are spatially associated with degenerating axons. ${ }^{26}$ Following contusion SCI in rats, apoptosis of oligodendrocytes is maximal 8 days postinjury. ${ }^{66}$ However, early activation of upstream and downstream apoptotis-inducing components has been reported within neurons following SCI. ${ }^{83}$ Emery et $a l^{80}$ confirmed the occurrence of apoptotic cells by caspase- 3 activation in human SCI $3 \mathrm{~h}$ to 2 months after injury. ${ }^{80}$ Rescuing oligodendrocytes and preservation of myelin are expected to have large effects on the functional outcome after SCI. ${ }^{16,63,89}$ Recently, using the anti-ssDNA monoclonal antibody F7-26 (Apostatin), it has been demonstrated that systemic administration of dexamethasone decreases apoptosis-related cell death in the injured spinal cord. ${ }^{90}$

Wallerian degeneration and demyelination Apoptosis of oligodendrocytes leads to chronic demyelination thus causing anterograde neurodegeneration. The degeneration of fibres characterized by the disruption of their myelin sheaths is known as Wallerian degeneration (WD). WD is accompanied by the activation of resident microglia that are in intimate spatial contact with apoptotic oligodendrocytes in the white matter, largely in ascending tracts above and in descending fibre tracts below the lesion in humans and rodents. ${ }^{16,66,80,82} \mathrm{WD}$ is partly responsible for the delayed sensory-motor dysfunction. Further, SCI induces a reduced expression of various myelin proteins; for example, proteolipid protein and myelin basic protein, at the transcriptional level. This causes abnormal myelination. ${ }^{91}$

\section{Regulation of CNS inflammation}

Intracellular signal transduction cascades regulate gene expression by converting transcriptional activator proteins from an inactive to an active state in which they are able to bind to DNA. ${ }^{92}$ A rich variety of signal transduction pathways exists within all neurons. Activation of these pathways is typically initiated by neurotransmitters that bind to receptors including ligandgated ion channels, G-protein-coupled receptors and tyrosine kinase receptors. A common outcome of the activation of these receptors is the production of second messengers such as cAMP and $\mathrm{Ca}^{2+}$, which bind to effector enzymes. Particularly important effectors are protein kinases and phosphatases that regulate the phosphorylation state of their substrates, and thus regulate their function. These substrates can be metabolic enzymes or signal transduction factors such as ion channels, protein kinases, or transcription factors that regulate gene expression.

Pro- and antiinflammatory cytokines tune this intracellular signal cascade. TNF- $\alpha$, IL- $1 \beta$, and IL-6 are considered to be proinflammatory cytotoxic cytokines and are critical mediators of the post-traumatic inflammatory reaction. IL-10 is a potent anti-inflammatory cytokine that supresses the majority of microglial/ macrophage responses. ${ }^{4,28,93}$ This cytokine blocks the activation of NF- $\kappa \mathrm{B}$ and attenuates the synthesis of various cytokines, chemokines, and matrixproteases. With respect to the CNS, IL-10 reduces the TNF- $\alpha$ production by astrocytes as well as the antigen presentation by both astrocytes and microglia. ${ }^{4,94}$ Further, IL-10 is known to act at the BBB preventing adhesion and extravasation of leucocytes. ${ }^{95}$ QUIS injection causes excitotoxic SCI. QUIS injection results in an increase mRNA encoding IL-1 $\beta$, cyclooxygenase-2 $\left(\right.$ Cox-2), and iNOS. ${ }^{28}$ QIUS injury in combination with systemic injection of IL-10 has been shown to significantly downregulate expressiom of IL- $1 \beta$ and iNOS mRNA. ${ }^{28}$ In mice lacking TNF- $\alpha$ receptors, reduced microglia activation was found following traumatic brain injury, ${ }^{96}$ suggesting that TNF- $\alpha$ plays an important role in microglia activation.

Intraspinal injection of recombinant IL- $1 \beta$ was found to induce significant BSB breakdown and recruitment of neutrophils and lymphocytes. In contrast, a similar injection into the brain parenchyma failed to evoke a BBB breakdown and neutrophil recruitment. ${ }^{10}$ Further, 
intracerebral microinjection of IL-1 $\beta$ was found to induce apoptosis of intrinsic CNS cells, whereas a similar injection of TNF- $\alpha$ did not cause DNA fragmentation. However, both caused vasogenic edema. ${ }^{97}$ Axotomy exerts strong IL-6 immunoreactivity in motoneurons and upregulation of IL-6 mRNA 2 days postinjury. This is claimed to trigger the proliferative aspect of microglia activation, thus having a neuroprotective function. 5,6,98 However, injection of fibroblasts secreting hyperinterleukin-6 (cytokine growth factor, IL-6, and its alpha receptor) did result in a marked increase in number of neutrophils and in the area occupied by activated microglia and macrophages, correlating with a two-fold increase in the lesion size. ${ }^{99}$

Following experimental SCI, transcripts of both the proinflammatory cytokines, TNF- $\alpha$, IL- $1 \beta$, and IL-6 and the chemokines, MIP- $1 \alpha$ and MIP- $1 \beta$, are upregulated within the first hour postinjury. ${ }^{100}$ However, the expression of $\mathrm{TNF}-\alpha$ is nearly completely downregulated by $24 \mathrm{~h} .^{2,5,57,101}$ Therefore, CNS cells, and not the invading non-CNS resident cells, seem to be the source of the transcripts. In juvenile rats, an age-related 'window of susceptibility' to the inflammatory stimulus on leucocytes and BBB permeability secondary to an intracerebral IL- $1 \beta$ injection has been demonstrated. ${ }^{102}$ Further, TNF- $\alpha$ prevents degradation of RGS7, a regulator of G-protein signalling, thus contributing to the regulation of CNS inflammation. ${ }^{103}$ SCI-induced expression of RGS7 in activated microglia and peripheral macrophages is supported by in vitro studies with TNF- $\alpha$-stimulated cell lines. ${ }^{36}$ This work suggests that a RGS7 modified G-protein regulates activation of microglia by $\mathrm{TNF}-\alpha$.

Plasma levels of inflammatory mediators, including cytokines (for example IL-2, IL-6), the soluble IL-2 receptor, and adhesion molecules (for example ICAM1), are elevated in chronic SCI patients. This may explain the prolonged inflammatory response in human SCI. ${ }^{18}$

Chondroitin Sulphate Proteoglycans (CSPs) are a group of inhibitory molecules of the injured CNS that are mainly produced by astrocytes. ${ }^{73}$ CSP within the extracellular matrix effectively encapsulate transplants of sensory neurons at the host-transplant interface. This prevents migration into the host white matter even in the absence of a physical scar barrier. ${ }^{35}$ The bacterial enzyme chondroitinase ABC (ChABC) trims the carbohydrate side chains off large extracellular proteins by degradation of CSP at the SCI site. It has been shown that intrathecal injection of ChABC yields an upregulation of a regeneration-associated protein in injured neurons and promotes regeneration in both ascending sensory projections and descending corticospinal tract axons. ${ }^{104}$ Further, ChABC treatment is also reported to restore postsynaptic activity below the lesion after electrical stimulation of corticospinal neurons and to promote functional recovery of locomotion and proprioception. $^{104}$

Proteinases, and in particular, matrix metalloproteinases (MMPs) are likely mediators of early secondary vascular pathogenesis after SCI. MMPs form a large family of zinc- and $\mathrm{Ca}^{2+}$-dependent endopeptidases that together can hydrolyse essentially all components of the extracellular matrix. ${ }^{39,105}$ MMPs are necessary for remodelling of the extracellular matrix. MMP activity is required for inflammatory cell infiltration and may contribute to the early alteration of the BSB permeability. MMP-9 is predominately expressed by inflammatory cells and plays an important role in BSB dysfunction and post-traumatic inflammation. MMP-9 is rapidly increased after contusion SCI and reaches a maximum at day 1. ${ }^{106}$ Methylprednisolone has been shown to suppress the expression of MMP-9 after SCI. ${ }^{107}$ Further, MMP released by axonal growth cones break down inhibitory CSPs. ${ }^{35}$

Whether or not inflammation is beneficial or deleterious depends not only on the cellular and soluble components but also, importantly, on the timing of the acute inflammatory process. Klusman and Schwab $^{6}$ showed that following SCI, the induction of a welltimed cytokine-specific inflammatory response in the spinal cord may be neuroprotective. ${ }^{6}$ They demonstrated that after injecting a cytokine cocktail (TNF- $\alpha$, IL-1 and IL-6) into the spinal cord 4 days after injury, there was a slight increase in the recruitment of peripheral macrophages while microglial activation was decreased. In contrast, with injection at day 1 after injury, an increase in the number of macrophages and microglial activation was observed. The balance between neuronal growth potential and inhibition by adult CNS environmental cues holds the key to structural and functional repair of the adult $\mathrm{CNS} .^{35}$

Interestingly, hypothermia attenuates the production of oxygen-free radicals, suppresses release of EAA, reduces intracellular $\mathrm{Ca}^{2+}$ overload, delays induction of iNOS, and diminishes induction of IL-1 $\beta$ messenger RNA. This finally inhibits the effects of the secondary injury by reducing the post-traumatic inflammation and lipid peroxidation. ${ }^{108}$ Therefore, moderate hypothermia may be used to beneficially modulate the cascade of the secondary injury.

The extensive repair following CNS injury in lower vertebrates may not be because of differences in CNS myelin structure alone. Since frogs and fish exhibit continual and indeterminate body growth throughout life, many extrinsic and intrinsic CNS conditions required for axonal outgrowth may endure and facilitate axonal regeneration. ${ }^{109}$ Differences in the regulation of post-traumatic inflammatory responses in lower vertebrates may protect them from further deterioration by the secondary injury.

\section{Summary}

SCI initiates a robust immune response characterized in part by the synthesis of cytokines and chemokines and a coordinated infiltration of the injured area by peripheral leucocytes. SCI-induced inflammation may result in a further deterioration of functional outcome because of the development of scar tissue and necrosis or apoptosis 
of neurons and oligodendrocytes. However, posttraumatic inflammation has a dual role. Apart from deleterious effects, beneficial effects of the inflammatory response are evident. Initially, the post-traumatic inflammatory response is dominated by the recruitment of neutrophils, peaking at day 1 postinjury, and by breakdown of the BSB. Cell adhesion molecules are rapidly upregulated in the vascular endothelium. From day 2 onwards, blood-born macrophages and activated CNS resident microglia dominate the inflammatory response. Lymphocytes also appear, though in small numbers. Astrocytes react with upregulation of GFAP expression.

\section{Acknowledgements}

I thank Dr Katharine L Blaschuk of the Miami Project to Cure Paralysis, University of Miami School of Medicine, for review of this manuscript.

\section{References}

1 Acarin $\mathrm{L}$ et al. Neuronal, astroglial and microglial cytokine expression after an excitotoxic lesion in the immature rat brain. Eur J Neurosci 2000; 12: 3505-3520.

2 Bartholdi D, Schwab ME. Expression of pro-inflammatory cytokine and chemokine mRNA upon experimental spinal cord injury in mouse: an in situ hybridization study. Eur J Neurosci 1997; 9: 1422-1438.

3 Bethea JR et al. Traumatic spinal cord injury induces nuclear factor-kappaB activation. J Neurosci 1998; 18: 3251-3260.

4 Bethea JR et al. Systemically administrated interleukin-10 reduces tumor necrosis factor- $\alpha$ production and significantly improves functional recovery following traumatic spinal cord injury in rats. $J$ Neurotrauma 1999; 16: 851-863.

5 Hayashi $\mathrm{M}$ et al. Sequential mRNA expression for immediate early genes, cytokines, and neurotrophins in spinal cord injury. J Neurotrauma 2000; 17: 203-218.

6 Klusman I, Schwab ME. Effects of pro-inflammatory cytokines in experimental spinal cord injury. Brain Res 1997; 762: 173-184.

7 Popovich PG et al. Cellular inflammatory response after spinal cord injury in Sprague-Dawley and Lewis rats. $J$ Comp Neurol 1997; 377: 443-464.

8 Schwab ME, Bartholdi D. Degeneration and regeneration of axons in the lesioned spinal cord. Physiol Rev 1996; 76: 319-370.

9 Schnell L et al. Acute inflammatory responses to mechanical lesions in the CNS: differences between brain and spinal cord. Eur J Neurosci 1999a; 11: 3648-3658.

10 Schnell L et al. Cytokine-induced acute inflammation in the brain and spinal cord. J Neuropathol Exp Neurol 1999b; 55: 245-254.

11 Grimpe B, Silver J. The extracellular matrix in axon regeneration. Prog Brain Res 2002; 137: 333-349.

12 Jones LL, Tuszynski MH. Spinal cord injury expression of keratan sulfate proteoglycans by macrophages, reactive microglia, and oligodendrocyte progenitors. J Neurosci 2002; 22: 4611-4624.
13 Zhang $\mathrm{Y}$ et al. Tenascin-C expression and axonal sprouting following injury to the spinal dorsal columns in the adult rat. $J$ Neurosci Res 1997; 49: 433-450.

14 Popovich PG. Immunonological regulation of neuronal degeneration and regeneration in the injured spinal cord. Prog Brain Res 2000; 128: 43-58.

15 Beattie MS, Bresnahan JC. Cell death, repair, and recovery of function after spinal cord injury in rats. In: Kalb RG, Strittmatter SM (eds). Neurobiology of Spinal Cord Injury. Humana Press Inc.: Totowa, NJ 2000.

16 Liu XZ et al. Neuronal and glial apoptosis after traumatic spinal cord injury. J Neuosci 1997; 17: 5395-5306.

17 Marmarou A et al. Traumatic brain tissue acidosis: experimental and clinical studies. Acta Neurochir Suppl 1993; 57: 160-164.

18 Segal JL et al. Circulating levels of IL-2R, ICAM-1, and IL-6 in spinal cord injuries. Arch Phys Med Rehabil 1997; 78: 44-47.

19 Beattie MS et al. Cell death and plasticity after experimental spinal cord injury. Prog Brain Res 2000; 128: 9-21.

20 Basso DM et al. Graded histological and locomotor outcomes after spinal cord contusion using the NYU weight-drop device versus transsection. Exp Neurol 1996; 139: $244-256$.

21 Basso DM et al. MASCIS evaluation of open field locomotor scores: effects of experience and teamwork reliability. J Neurotrauma 1996; 13: 343-359.

22 Constantini S, Young W. The effects of methylprednisolone and the ganglioside GM1 on acute spinal cord injury in rats. $J$ Neurosurg 1994; 80: 97-111.

23 Gruner JA. A monitored contusion model of spinal cord injury in the rat. J Neurotrauma 1992; 9: 123-128.

24 Behrmann DL et al. Spinal cord injury produced by consistent mechanical displacement of the cord in rats: behavioral and histologic analysis. J Neurotrauma 1992; 9: 197-217.

25 Bresnahan JC. A behavioral and anatomical analysis of spinal cord injury produced by a feedback-controlled impaction device. Exp Neurol 1987; 95: 548-570.

26 Casha $\mathrm{S}$ et al. Oligodendroglial apoptosis occurs along degenerating axons and is associated with Fas and p75 expression following spinal cord injury in the rat. Neuroscience 2001; 103: 203-218.

27 Abraham KE et al. The role of kainic acid/AMPA and metabotropic glutamate receptors in the regulation of opiod mRNA expression and the onset of pain-related behaviour following excitotoxic spinal cord injury. Neuroscience 2001; 104: 836-874.

28 Plunkett $\mathrm{J}$ et al. Effects of interleukin-10 on pain behavior and gene expression following excititoxic spinal cord injury in the rat. Exp Neural 2001; 168: 144-154.

29 Beattie MS et al. ProNGF induces p75-mediated death of oligodendrocytes following spinal cord injury. Neuron 2002; 36: 375-386.

30 Joshi M, Fehlings M. Development and characterization of a novel, graded model of clip compressive spinal cord injury in the mouse: Part 1. Clip design, behavioral outcome, and histopathology. J Neurotrauma 2002; 19: $175-190$.

31 Joshi M, Fehlings M. Development and characterization of a novel, graded model of clip compressive spinal cord injury in the mouse: Part 2. Quantitative neuroanatomical assessment and analysis of the relationship between axonal tracts, residual tissue, and locomotor recovery. $J$ Neurotrauma 2002; 19: 191-203. 
32 Stokes BT, Jakeman LB. Experimental modeling of human spinal cord injury: a model that crosses the species barrier and mimics the spectrum of human cytopathology. Spinal Cord 2002; 40: 101-109.

33 Beal MF. Energetics in the pathogenesis of neurodegenerative diseases. Trends Neurosci 2000; 23: 298-304.

34 Beattie MS et al. Cell death in models of spinal cord injury. Prog Brain Res 2002; 137: 37-47.

35 Davies SJ, Silver J. Adult axon regeneration in adult CNS white matter. Trends Neurosci 1998; 21: 515.

36 Hausmann ON et al. Spinal cord injury induces expression of RGS7 in microglia/macrophages in rats. Eur $J$ Neurosci 2002; 15: 602-612.

37 Noble LJ et al. Disruption and time course of protein extravasation in the rat spinal cord after contusive injury. Brain Res 1989; 482: 57-66.

38 Pan W, Kastin AJ. Increase in TNFalpha transport after $\mathrm{SCI}$ is specific for time, region, and type of lesion. Exp Neurol 2001; 170: 357-363.

39 Mautes AE et al. Vascular events after spinal cord injury: contribution to secondary pathogenesis. Phys Ther 2000; 80: 673-687.

40 Tator $\mathrm{CH}$, Koyanagi I. Vascular mechanisms in the pathophysiology of human spinal cord injury. $J$ Neurosurg 1997; 86: 483-492.

41 Saikumar $\mathrm{P}$ et al. Mechanisms of cell death in hypoxia/ reoxygenation injury. Oncogene 1998; 17: 3341-3349.

42 Mills CD et al. Changes in metabotropic glutamate receptor expression following spinal cord injury. Exp Neurol 2001; 170: 244-257.

$43 \mathrm{Ha}$ BK et al. Kainate-induced excitotoxicity is dependent upon extracellular potassium concentrations that regulate the activity of AMPA/KA type receptors. J Neurochem 2002; 83: 934-945.

44 Mills CD et al. Involvement of metabotropic glutamate receptors in excitatory amino acid and GABA release following spinal cord injury in rat. $J$ Neurochem 2001; 79: $835-848$

$45 \mathrm{Chu}$ GK et al. Calcium and neuronal death in spinal neurons. In: Kalb RG, Strittmatter SM (eds). Neurobiology of Spinal Cord Injury. Humana Press Inc.: Totowa, NJ 2000.

46 Regan RF, Choi DW. Glutamate neurotoxicity in spinal cord cell culture. Neuroscience 1991; 43: 585-591.

47 Vera-Portocarrero LP et al. Rapid changes in expression of glutamate transporters after spinal cord injury. Brain Res 2002; 927: 104-110.

48 Regan RF. The vulnerability of spinal cord neurons to excitotoxic injury: comparison with cortical neurons. Neurosci Lett 1996; 213: 9-12.

49 Choi DW. Glutamate receptors and the induction of excitotoxic neuronal cell death. Curr Opin Neurobiol 1996; 6: $667-672$.

50 Jansco $\mathrm{G}$ et al. Neurotoxin induced nerve cell degeneration: possible involvement of calcium. Brain Res 1984; 295: $211-216$.

51 Aizenman E et al. Oxygen free radicals regulate NMDA receptor function via a redox modulatory site. Neuron 1990; 5: 841-846.

52 Schmidley JW. Free radicals in the central nervous system ischemia. Stroke 1990; 21: 1086-1090.

53 Dalkara $\mathrm{T}$ et al. Constitutive nitric oxide synthase and ischemic/excitotoxic brain injury. In: Ruffolo RR et al (eds). Inflammatory Cells and Mediators in CNS Diseases. New Academic Publishers: Amsterdam 1999.
54 Lipton SA et al. A redox-based mechanism for the neuroprotective and neurodestructive effects of nitric oxide and related nitroso-compounds. Nature 1993; 364: 626-631.

55 Nakahara $\mathrm{S}$ et al. Changes in nitric oxide and expression of nitric oxide synthase in spinal cord after traumatic injury in rats. $J$ Neurotrauma 2002; 11: 1467-1474.

56 Bao F, Liu D. Peroxynitrite generated in the rat spinal cord induces neuron death and neurological deficits. Neuroscience 2002; 115: 839-849.

57 Dawson VL et al. Mechanisms of nitric oxide-mediated neurotoxicity in primary cortical cultures. J Neurosci 1993; 13: 2651-2661.

58 Brewer GJ, Wallimann T. Protective effects of the energy precursor creatine against toxicity of glutamate and betaamyloid in rat hippocampal neurons. $J$ Neurochem 2000; 74: $1968-1978$.

59 Hausmann ON et al. Protective effects of oral creatine supplementation prior to spinal cord injury in rats. Spinal Cord 2002; 40: 449-456.

60 Taoka Y, Okajima K. Role of leucocytes in spinal cord injury in rats. J Neurotrauma 2000; 17: 219-229.

61 Carlson SL et al. Acute inflammatory response in spinal cord following impact injury. Exp Neurol 1998; 151: $77-88$.

62 Chatzipanteli $\mathrm{K}$ et al. Post-traumatic hypothermia reduces polymorphnuclear leucocyte accumulation following spinal cord injury in rats. $J$ Neurotrauma 2000; 17: 321-332.

63 Taoka Y, Okajima K: Spinal cord injury in the rat. Prog Neurobiol 1998; 56: 341-358.

64 Watanabe $\mathrm{T}$ et al. Differential activation of microglia after experimental spinal cord injury. $J$ Neurotrauma 1999; 16: 255-265.

65 Streit WJ et al. Functional plasticity of microglia: a review. Glia 1988; 1: 301-307.

66 Shuman SL et al. Apoptosis of microglia and oligodendrocytes after spinal cord injury in rats. $J$ Neurosci Res 1997; 50: 798-808.

67 Schwartz M. Autoimmune involvement in CNS trauma is beneficial if well controlled. Prog Brain Res 2000; 128: 259-263.

68 Rapalino O et al. Implantation of stimulated homologous macrophages results in partial recovery of paraplegic rats. Nat Med 1998; 4: 814-821.

69 Hauben $\mathrm{E}$ et al. Autoimmune $\mathrm{T}$ cells as potential neuroprotective therapy for spinal cord injury. Lancet 2000; 354: 286-287.

70 Nevo U et al. Diffusion anisotrophy MRI for quantitative assessment of recovery in injured rat spinal cord. Magn Reson Med 2001; 45: 1-9.

71 Stichel CC, Müller HW. The CNS lesion scar: new vistas on an old regeneration barrier. Cell Tissue Res 1998; 249: $1-9$.

72 Fawcett JW. Spinal cord repair: from experimental models to human application. Spinal Cord 1998; 36: 811-817.

73 Fawcett JW, Asher RA. The glial scar and central nervous system repair. Brain Res Bull 1999; 49: 377-391.

74 Fitch MT, Silver J. Glial cell extracellular matrix: boundaries for axon growth in the development and regeneration. Cell Tissue Res 1997a; 290: 379-384.

75 Goss JR. Astrocytes are the major source nerve growth factor upregulation following traumatic brain injury in the rat. Exp Neurol 1998; 149: 301-309. 
76 Liesi $\mathrm{P}$ et al. Laminin is induced in astrocytes of the adult brain by injury. EMBO $J$ 1984; 3: 683-686.

77 Davies SJ et al. Regeneration of adult axons in white matter tracts of the central nervous system. Nature 1997; 390: 680-683.

78 Davies SJ et al. Robust regeneration of adult sensory axons in degenerating white matter of the adult rat spinal cord. J Neurosci 1999; 19: 5810-5822.

79 McGraw $\mathbf{J}$ et al. Modulating astrogliosis after neurotrauma. J Neurosci Res 2001; 63: 109-115.

80 Emery E et al. Apoptosis after traumatic human spinal cord injury. J Neurosurg 1998; 89: 911-920.

81 Majno $\mathrm{G}$ et al. Apoptosis, oncosis, and necrosis. An overview of cell death. Am J Pathol 1995; 146: 3-15.

82 Crowe MJ et al. Apoptosis and delayed degeneration after spinal cord injury in rats and monkeys. Nat Med 1997; 3: 73-76.

83 Springer JE et al. Activation of the caspase-3 apoptotic cascade in traumatic spinal cord injury. Nat Med 1999; 5: 943-946.

84 Baker SJ, Reddy EP. Modulation of life and death by the TNF receptor superfamily. Oncogene 1998; 17: 3261-3270.

85 Li GL et al. Changes of Fas and Fas ligand immunoreactivity after compression trauma to rat spinal cord. Acta Neuropathol 2000; 100: 75-81.

86 Zurita M et al. Presence and significance of CD-95 (Fas/ APO1) expression after spinal cord injury. $J$ Neurosurg (Spine) 2001; 94: 257-264.

87 Lee YB et al. Role of tumor necrosis factor- $\alpha$ in neuronal and glial apoptosis after spinal cord injury. Exp Neurol 2000; 166: 190-195.

88 Wada S. Apoptosis following spinal cord injury in rats and preventative effects of $N$-methyl-D-aspartate receptor agonist. J Neurosurg (Spine 1) 1999; 91: 98-104.

89 Rabchevsky AG et al. Basic fibroblast growth factor (bFGF) enhances tissue sparing and functional recovery following moderate spinal cord injury. $J$ Neurotrauma 1999; 16: 817-830.

90 Zurita $\mathrm{M}$ et al. Effects of dexamethasone on apoptosisrelated cell death after spinal cord injury. $J$ Neurosurg (Spine) 2002; 96: 83-89.

91 Wrathall JR et al. Myelin gene expression after experimental contusive spinal cord injury. J Neurosci 1998; 18: 8780-8793.

92 Purves $\mathrm{D}$ et al. Intracellular signal transduction. In: Purves D et al (eds). Neuroscience. 2nd ed. Sinauer Associates: Sunderland, MA 2001.

93 O'Farrell AM et al. IL-10 inhibits macrophage activation and proliferation by distinct signalling mechanisms: evidence for Stat3-dependent and -independent pathways. EMBO J 1998; 17: 1006-1013.

94 Dietrich WD et al. Postischemic hypothermia and IL-10 treatment provide long-lasting neuroprotection of CA1 hippocampus following transient global ischemia in rats. Exp Neurol 1999; 158: 444-450.

95 Knoblauch SM et al. Interleukin-10 improves outcome and alters proinflammatory cytokine expression after experimental traumatic brain injury. Exp Neurol 1998; 153: $143-151$.

96 Bruce AJ et al. Alterated neuronal and microglial responses to excitotoxic and ischemic brain injury in mice lacking TNF receptors. Nat Med 1996; 2: 788-794.
97 Holmin S et al. Intracerebral administration of interleukin-1b and induction of inflammation, apoptosis, and vasogenic edema. J Neurosurg 2000, 92: 108-120.

98 Streit WJ et al. Comparative evaluation of cytokine profiles and reactive gliosis supports a critical role for interleukin-6 in neuron-glia signaling during regeneration. J Neurosci Res 2000; 61: 10-20.

99 Lacroix S et al. Delivery of hyper-interleukin-6 to the injured signal cord increases neutrophil and macrophage infiltration and inhibits axonal growth. J Comp Neurol 2002; 454: 213-228.

100 Pan JZ et al. Cytokine activity contributes to induction of inflammatory cytokine mRNAs in spinal cord following contusion. J Neurosci Res 2002; 68: 315-322.

101 Wang CX et al. Increase of interleukin-1B mRNA and protein in the spinal cord following experimental traumatic injury in the rat. Brain Res 1997; 759: 190-196.

102 Anthony DC et al. Age-related effects of interleukin 1B on polymorphnuclear neutrophil-dependent increases in blood-brain barrier permeability in rats. Brain 1997; 120: 435-444.

103 Benzing T et al. Upregulation of RGS7 may contribute to tumor necrosis factor induced changes in central nervous function. Nat Med 1999; 5: 913-918.

104 Bradburry EJ et al. Chondroitinase ABC promotes functional recovery after spinal cord injury. Nature 2002; 416: 636-640.

105 Birkedal-Hansen $\mathrm{H}$ et al. Matrix metalloproteases: a review. Crit Rev Oral Biol Med 1993; 4: 197-250.

106 Noble LJ et al. Matrix metalloproteases limit functional recovery after spinal cord injury by modulation of early vascular events. $J$ Neurosci 2002; 22: 7526-7535.

$107 \mathrm{Xu} \mathbf{J}$ et al. Glucocorticoid receptor-mediated suppression of activator protein-1 activation and metalloproteinase expression after spinal cord injury. J Neurosci 2001; 2: $502-511$.

108 Guang C et al. Beneficial effects of modest hypothermia on locomotor function and histopathological damage following contusion-induced spinal cord injury in rats. J Neurosurg (Spine1) 2000; 93: 85-93.

109 Stevens JD, Tetzlaff W. Strategies for spinal cord repair. In: Kalb RG, Strittmatter SM (eds). Neurobiology of Spinal Cord Injury. Humana Press Inc.: Totowa, NJ 2000.

110 Behrmann DL et al. Modeling spinal cord injury in the rat: neuroprotection and enhanced recovery with methylprednisolone and YM-14673. Exp Neurol 1994; 126: $61-75$.

111 Fitch MT, Silver J. Activated macrophages and the blood-brain barrier: inflammation after CNS injury leads to increase in putative molecules. Exp Neurol 1997b; 148: 587-603.

112 Krautstrunk M et al. Increased expression of the putative axon growth-repulsive extracellular matrix molecule, keratan sulphate proteoglycan, following traumatic injury of the adult rat spinal cord. Acta Neuropathol 2002; 104: 592-600.

113 Schwartz M. Protective autoimmunity as a T-cell response to central nervous system trauma: prospects for therapeutic vaccines. Prog Neurobiol 2001; 65: 489-496.

114 Yakolev AG et al. Activation of CPP32-like caspases contributes to neuronal apoptosis and neurological dysfunction after traumatic brain injury. $J$ Neurosci 1997; 17: 7415-7424. 